PlastOx 2007 (2009) 71-80

(C) EDP Sciences, 2009

DOI: $10.1051 /$ ptox/2009007

\title{
Lacunes surabondantes dans les systèmes métal-hydrogène et endommagement des joints de grains : simulations atomiques
}

\author{
D. Tanguy ${ }^{1}$ et E. Vamvakopoulos ${ }^{2}$ \\ ${ }^{1}$ CNRS, UMR 5146, École des Mines de Saint-Étienne, 158 cours Fauriel, \\ 42023 Saint-Étienne, France \\ ${ }^{2}$ Centre Européen de Calcul Atomique et Moléculaire, École Normale Supérieure de Lyon, \\ 46 allée d'Italie, 69007 Lyon, France
}

\begin{abstract}
Résumé. Ce travail porte sur la modélisation de la perte de cohésion des joints de grains dans $\mathrm{Al}$ par ségrégation massive d'hydrogène $(\mathrm{H})$. Nous tentons de poser simplement ce problème dans le contexte très " multi-échelle » de l'endommagement par $\mathrm{H}$ de matériaux ductiles : les alliages métalliques cubiques à faces centrées. En se référant aux calculs ab initio de la littérature, il est montré que la ségrégation de $\mathrm{H}$ seul ne permet pas de rendre compte de la décohésion observée expérimentalement. Un ingrédient microscopique supplémentaire doit être considéré. Nous proposons de prendre en compte la formation de lacunes surabondantes au niveau des joints (reconstruction des joints induite par $\mathrm{H}$ ). Pour tester cette hypothèse, des méthodes de simulation à l'échelle atomique sont développées : Monte Carlo, pour le problème des lacunes dans le joint, et Dynamique Moléculaire contrainte, pour la propagation de fissures.
\end{abstract}

\section{INTRODUCTION}

Ce travail contribue à la modélisation de l'endommagement par l'hydrogène des joints de grains des alliages métalliques dits « de structure » (bases Ni, aciers, alliages Al-Mg (5XXX), Al-Zn-Mg (7XXX), etc.). Le phénomène est complexe car il fait intervenir de nombreux mécanismes microscopiques à des échelles de temps et d'espace différentes [1]. Faisons une rapide liste des principaux mécanismes (Fig. 1) :

- Il y a tout d'abord la production par les réactions électrochimiques en milieu aqueux et l'entrée de l'hydrogène en surface, ou à partir des lèvres d'une fissure de corrosion sous contrainte $[2,3]$. Les mécanismes de corrosion en milieux confinés (fond de fissure) sont très complexes à modéliser et à étudier expérimentalement.

- L'hydrogène diffuse depuis les lèvres vers la pointe (adsorption en pointe de fissure) ou en avant de celle-ci. Il s'établit un profile de concentration (hors équilibre), en avant de la pointe, qui dépend d'effets élastiques (champ de contrainte de la fissure chargée mécaniquement, champs des dislocations de la zone plastique) et de la microstructure (piégeage au niveau des précipités intra et intergranulaires...).

- L'hydrogène, en interstitiel dans le réseau, modifie les interactions élastiques entre les dislocations $[4,5]$, ce qui influence la formation de la zone plastique, donc l'écrantage de la fissure et donc les mécanismes (ou au moins les seuils) de propagation de fissures (pour une revue, voir Ref. [6]).

Les simulations à l'échelle atomique, qui sont limitées en temps (quelques centaines de nanosecondes) et en taille $(40 \mathrm{~nm} * 40 \mathrm{~nm} * 40 \mathrm{~nm}$ représente déjà plusieurs millions de particules), ne sont évidemment pas bien adaptées à tous ces problèmes, mais peuvent apporter des éclairages décisifs sur certains points. La figure 1 représente schématiquement les deux sous-problèmes, simples, qui seront détaillés dans cet article : le piégeage de l'hydrogène au niveau des interfaces et son influence sur la structure de la pointe de la fissure. Plus précisément, nous étudions la capacité de la pointe à émettre la première dislocation, en compétition avec la propagation purement fragile de la fissure. Ce qu'on appelle la fragilité/ductilité intrinsèque du matériau. 


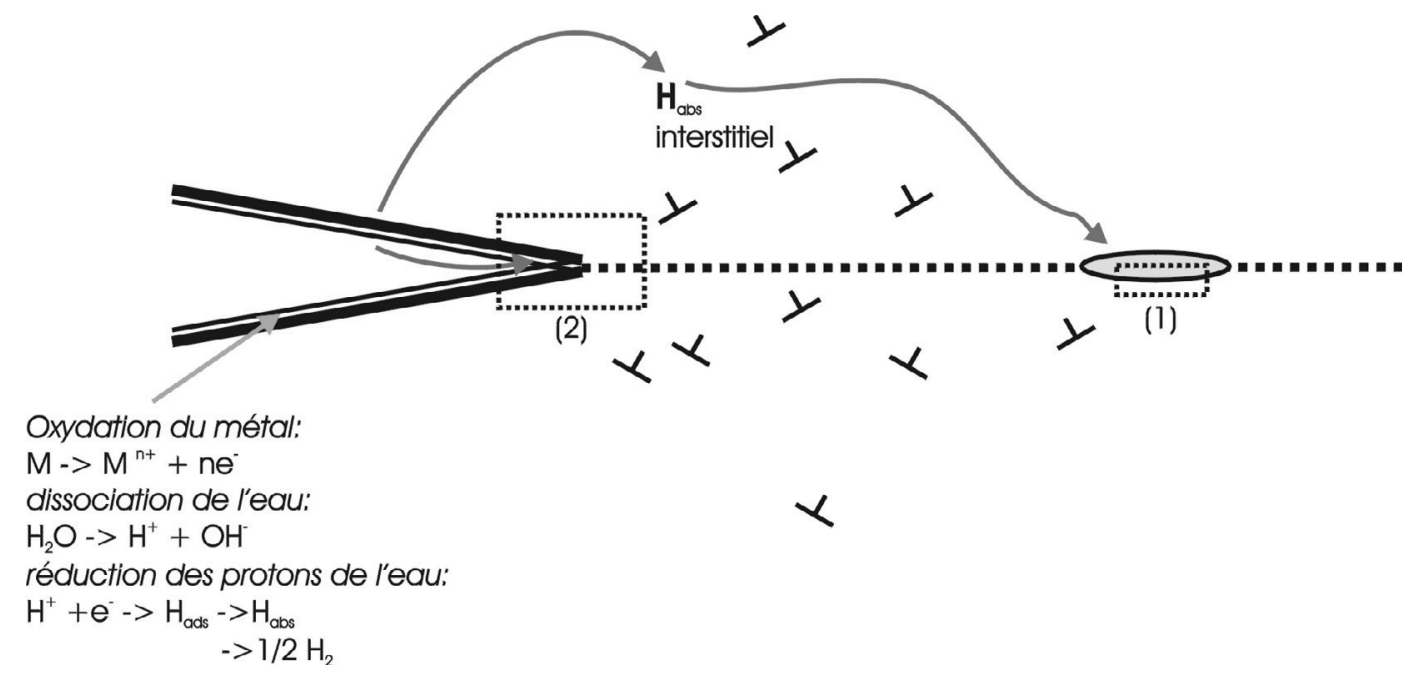

Figure 1. Représentation schématique des principaux mécanismes amenant à l'endommagement par l'hydrogène. Sous-problèmes traités à l'échelle atomique : (1) piégeage de $\mathrm{H}$ aux interfaces (traité comme une ségrégation d'équilibre à une concentration locale donnée par le profile de diffusion); (2) analyse fine de la pointe de la fissure, calcul de $\mathrm{k}_{\mathrm{Ic}}$ et $\mathrm{k}_{\mathrm{Ie}}$ pour déterminer la fragilité/ductilité intrinsèque du matériau.

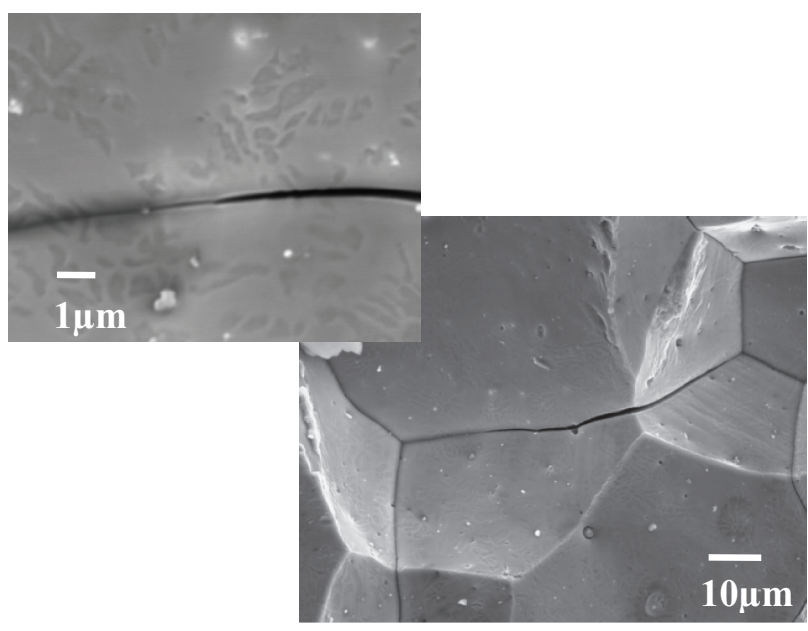

(a)

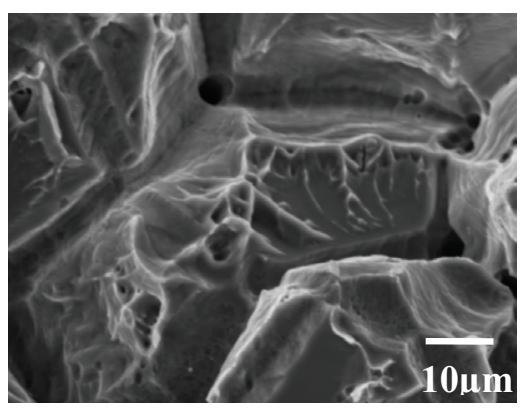

(b)

Figure 2. Faciès de rupture obtenus sur $\mathrm{Al}-5 \% \mathrm{Mg}$ (a) dans $\mathrm{AlCl}_{3}$ dés oxygéné en corrosion sous contrainte, (b) sur un échantillon fragilisé par $\mathrm{H}$, puis soumis à un traitement de désorption. Le faciès après fragilisation est analogue à (a). Après désorption, la ductilité est partiellement restaurée, comme le montre les nombreux ligaments ductiles sur (b). On note que la rupture a quand même lieu en suivant les joints de grains.

L'influence de $\mathrm{H}$ sur la rupture peut être spectaculaire, comme le montre les observations de faciès en microscopie à balayage (MEB) : des matériaux ductiles à l'air, comme les alliages d'aluminium, peuvent présenter une certaine fragilité intergranulaire (Fig. 2). Cependant, les mécanismes microscopiques ne sont pas établis, même s'il y a une bonne intuition de ce qui peut se passer. Deux mécanismes principaux sont invoqués [6] : une baisse de la cohésion, surtout aux interfaces, où les sites de piégeage sont naturellement présents tout le long du chemin de propagation; une localisation et une intensification 
de la déformation plastique par adsorption de $\mathrm{H}$ à la pointe (émission de dislocations facilitée depuis la pointe) et adoucissement en avant de pointe par écrantage des interactions élastiques dislocationdislocation (nécessite de fortes concentrations locales, de l'ordre quelques pour cent) [7, 8]. Le HELP («Hydrogen Enhanced Localized Plastity ») est, à lors actuel, le modèle de rupture transgranulaire reconnu. Les plages planes à l'échelle sur les faciès de rupture, qui pourraient être interprétées comme de la fragilisation, proviendraient de cisaillements plans dans des bandes de glissement intenses.

Pour pouvoir avancer plus avant dans la modélisation de l'endommagement, il faut reprendre un par un les mécanismes élémentaires et essayer de quantifier le rôle de H. L'objectif de notre travail, à terme, est de clarifier l'effet de H sur la cohésion des joints de grains. Ceci n'est que l'un des éléments qui entre dans la modélisation de la rupture, car, dans les matériaux de structure qui nous intéressent, la propagation fragile est toujours accompagnée de déformation plastique.

L'article est organisé en trois parties. Tout d'abord, les limitations des simulations de décohésion actuelles sont exposées. Nous montrons que les «lacunes surabondantes » (Super Abundant Vacancies » SAV) peuvent être un acteur essentiel dans la chute de cohésion des interfaces. Ensuite, les simulations de SAV par méthode Monte Carlo sont présentées, dans le cristal parfait, et, en perspectives, dans le joint de flexion symétrique $\Sigma=33$ (554) [110]. Enfin, des simulations par Dynamique Moléculaire de propagation de fissures, dans un monocristal sollicité dans la direction [111], sont détaillées. Cette méthode permet de calculer $\mathrm{k}_{\mathrm{Ic}}$ (le facteur d'intensité de contrainte critique pour la propagation fragile) et $\mathrm{k}_{\mathrm{Ie}}\left(\mathrm{k}_{\mathrm{I}}\right.$ critique pour l'émission de la première dislocation partielle depuis la pointe de la fissure). Le modèle de Rice et Thomson [9] donne une première idée du comportement ductile/fragile d'un matériau en comparant ces deux valeurs. Connaître l'influence de $\mathrm{H}$ sur ces grandeurs constitue une première étape vers la modélisation des effets de l'hydrogène sur la rupture. Influence qui se manifestera sur l'émission de dislocations (HELP) et sur les propriétés de cohésion.

\section{DÉCOHÉSION ET LACUNES SURABONDANTES}

L'influence de $\mathrm{H}$ sur la cohésion d'un monocristal sollicité dans la direction (111) a été calculée récemment en ab initio dans $\mathrm{Al}[10,11]$. La simulation consiste à insérer une concentration d'hydrogène définie, en position interstitielle dans le réseau. Le cristal est ensuite séparé en deux blocs et les $\mathrm{H}$ placés en surface (diffusion instantanée). Le travail idéal de rupture est la différence entre l'énergie de la configuration «collée » et la configuration complètement « séparée », i.e. celle où les surfaces sont suffisamment éloignées pour ne plus interagir et où $\mathrm{H}$ se retrouve adsorbé en surface. Si on néglige les interactions $\mathrm{H}-\mathrm{H}$, dans le volume et en surface, on peut écrire que :

$$
2 \gamma\left(\theta_{H}\right)=2 \gamma_{0}+2 \theta_{H} \Delta E_{\text {seg }}
$$

où $2 \gamma\left(\theta_{H}\right)$ est le travail idéal de rupture, $2 \gamma_{0}$ est l'énergie d'excès des deux surfaces crées sans $\mathrm{H}, \Delta E_{\text {seg }}$ l'énergie de ségrégation de surface de $\mathrm{H}$ et $\theta_{H}$ la concentration en hydrogène sur la surface. La variation du travail idéal de rupture avec la concentration est quasiment linéaire. Pour un taux de couverture de $100 \%$ soit une mono-couche (MC) (un atome $\mathrm{H}$ pour un atome $\mathrm{Al}$ ), $2 \gamma\left(\theta_{H}\right)$ chute spectaculairement et ne vaut plus que $33 \%$ de sa valeur sans $\mathrm{H}$ pour $\mathrm{Al}(111)$ (et $19 \%$ pour $\mathrm{Fe}(110)$, pour comparaison). Si on injecte cette valeur dans le critère de Griffith $\left(k_{I c}=\alpha \sqrt{\gamma}\right)$, pour la propagation par décohésion pure, on trouve $k_{I c}(1 M C)=0.58 k_{I c}(0 M C)$. Il est difficile de se rendre compte de l'impact de cette chute sans un modèle mésoscopique qui prenne en entrée ces valeurs et simule une propagation de fissure [12] avec relaxation plastique. Ces calculs sont néanmoins très utiles puisqu'ils offrent des données sur lesquelles ajuster des potentiels interatomiques empiriques qui permettent de faire des calculs sur des systèmes, plus grands, comportant des défauts cristallins (joints de grains, fissures), comme montré par la suite.

Les calculs sur des configurations monocristallines sont ceux qui montrent le maximum d'effet de $\mathrm{H}$ sur le travail de rupture car les interstitiels passent des sites de volume aux sites de surface, où ils 
gagnent le maximum d'énergie. C'est donc une borne supérieure de la chute de cohésion. Néanmoins, il n'a jamais été observé expérimentalement que l'hydrogène adsorbé suffise à forcer la propagation transgranulaire fragile. En général, une déformation plastique intense est observée. Il est plus aisé de chercher des pertes de cohésion quand l'hydrogène est absorbé et se trouve piégé aux joints de grains.

Quels sont les résultats expérimentaux ? A notre connaissance, Al pur n'a jamais été fragilisé au delà du premier micron sous la surface. Par contre, les alliages présentant des précipités intergranulaires le sont. L'effet est particulièrement frappant dans les alliages à durcissement structural de la famille 7XXX (Al-ZnMg bas $\mathrm{Cu}$ ). De nombreux auteurs [13-17] ont rapporté la formation de bulles d'hydrogène, à l'interface précipité intergranulaire/matrice après corrosion généralisée lente de la surface ou après piqûration des joints de grains. L'interprétation est la suivante : l'hydrogène, produit par les réactions cathodiques de surface, pénètre préférentiellement aux joints (surtout s'ils comportent des précipités riches en $\mathrm{Mg}$ ). L'émergence des précipités en surface constitue un «point faible » dans le film d'oxyde. Sans précipités, le film passif de l'aluminium constitue une barrière efficace contre l'entrée d'hydrogène. Les lames minces, prélevées sur des échantillons pré-corrodés, sont irradiées par le faisceau MET durant les observations. Ceci favorise la formation de lacunes qui se stabilisent en piégeant H. Ces complexes lacune-hydrogène s'agrègent pour former des bulles nanométriques. Sur les images MET seul le contraste associé au champ de contrainte, beaucoup plus large que les bulles elles mêmes, est visible. La croissance des bulles est suivie in situ. La fissuration fragile le long des joints de grains peut intervenir sans l'application de contraintes extérieures. En général la présence de bulles n'est pas nécessaire à la fragilisation (H piégé aux interfaces est suffisant). C'est l'un des rares cas, à notre connaissance, où des rupture purement fragiles, par effet d'hydrogène, ont été observées directement. Le rôle joué par les précipités intergranulaires n'est pas clair : on sait qu'ils permettent l'entrée de l'hydrogène dans le matériau; par contre sont-ils vraiment des sites de piégeage nécessaires ? Autrement dit, une fois l'hydrogène dans le volume, est-ce que le piégeage sur des joints de grains «propres » est suffisant pour faire chuter la cohésion?

Les calculs d'énergies de ségrégation intergranulaires permettent de se faire une idée de la baisse du travail idéal de rupture qui peut être attendue après ségrégation [18]. La situation qui est considérée dans ce paragraphe n'est pas la même que celle concernant les plans $\{111\}$. Cette fois, l'hydrogène est piégé à l'interface avant décohésion et on suppose qu'il n'y a pas de diffusion des sites de volume vers les surfaces nouvellement créées (Fig. 3 trajet c-d). Autrement dit, tout l'hydrogène présent dans le joint se retrouve en surface. Sans hydrogène, le travail idéal de rupture est la différence entre l'énergie de formation des surfaces et l'énergie d'excès du joint (Fig. 3 trajet a-b). On suppose qu'il est du même ordre de grandeur que l'énergie de formation de surfaces $\{111\}$ (surfaces denses, de faible énergie de formation). S'il y a $\theta_{H}$ hydrogènes dans le joint, l'énergie que gagne le système quand les $H$ passe du joint en surface est la différence entre l'énergie d'adsorption et l'énergie de ségrégation dans le joint : $W\left(\theta_{H}\right)=2 \gamma_{0}-\gamma_{\text {joint }}+\theta_{H} \times\left(\Delta E_{\text {ads }}-\Delta E_{\text {seg }}^{\text {joint }}\right)$. Or, les sites de ségrégation intergranulaires offrent une distribution d'énergies allant de zéro à l'énergie d'adsorption. La chute du travail idéal de rupture sera donc plus faible que dans le cas des surfaces $\{111\}$. Plus important : la baisse de W dépend de $\theta_{H}$ donc, si la densité de sites de ségrégation est faible, la chute de $\mathrm{W}$ est faible... Ce qui est le cas quand on considère des structures de joints de faible énergie [18]. $\theta_{H}$ est très loin d'atteindre la monocouche. Il faut donc rajouter un ingrédient microscopique supplémentaire pour arriver à chutes de cohésion qui permettraient aux joints de casser sous des charges très faibles, comme ce qui est observé expérimentalement.

La communauté a longtemps pensé aux lacunes produites par la dissolution anodique de surface. Les observations expérimentales, en pointe de fissure, étant difficiles, l'idée n'a pas débouché sur un modèle quantitatif. Il y a eu un regain d'intérêt pour les interactions hydrogène-lacune, suite à la découverte par Y. Fukai des « lacunes surabondantes ». Sous haute pression de gaz $\mathrm{H}_{2}$, à haute température, les concentrations d'équilibre de lacunes sont plusieurs ordres de grandeur plus élevées qu'en milieu neutre. La raison est la formation de clusters lacune-hydrogène. L'énergie de ségrégation de $\mathrm{H}$ sur une lacune est négative. De plus, elle peut accueillir plusieurs H (occupation multiple). L'énergie de formation de ces 


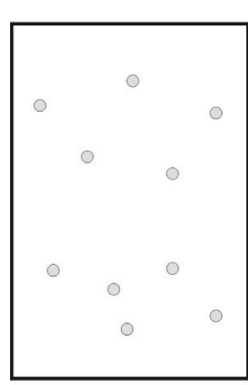

Référence
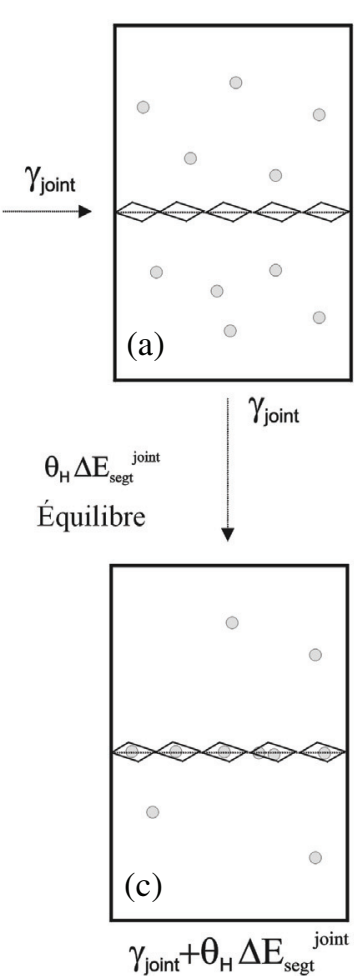

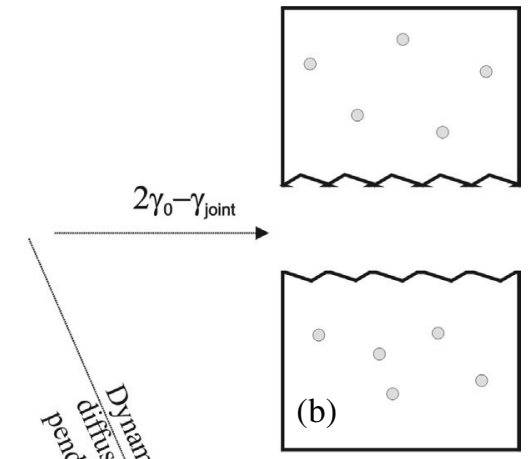

$2 \gamma_{0}$

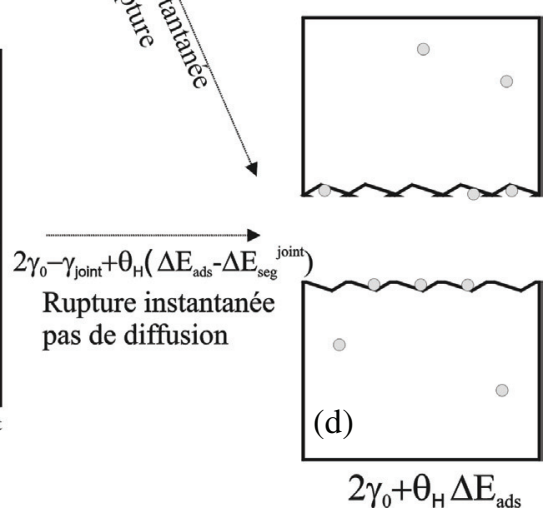

Figure 3. Représentation schématique des chemins «thermodynamiques » pour la décohésion. L'état de référence est le cristal parfait avec une concentration fixe d'hydrogène en interstitiel. Trajet $(a-b)$ : rupture d'un joint de grain « propre »; trajet $(\mathrm{a}-\mathrm{d})$ rupture avec diffusion instantanée d'une quantité $\theta_{\mathrm{H}}$ d'hydrogène ; trajet $(\mathrm{c}-\mathrm{d})$ rupture d'un joint de grain comportant une ségrégation d'hydrogène initiale, sans diffusion depuis les sites de volume adjacents. C'est le cas le plus réaliste pour les alliages d' $\mathrm{Al}$ en corrosion sous contrainte où les concentrations sont très faibles dans le volume. Il y a donc peu d'opportunité de diffuser vers le joint pendant la propagation de la fissure.

clusters devient négative (l'état de référence étant $\mathrm{H}$ en interstitiel dans le volume). Ce phénomène est observé dans une multitude de métaux $\mathrm{Pd}, \mathrm{Cu}, \mathrm{Fe}, \mathrm{Ni}$... Pour une revue, le lecteur pourra se référer à [19] et [20]. Ce qui est intéressant est que les hautes températures ne sont nécessaires que pour des raisons de cinétique de formation de lacunes. Ces clusters sont stables à l'ambiante. Dans Al pur [21-23], chargé électrochimiquement dans des conditions proches de celles rencontrées en corrosion sous contrainte, il a été montré que $\mathrm{H}$ diffuse depuis la surface sous forme de clusters lacune- $1 \mathrm{H}$. Ils peuvent se recombiner (ou être recombinés par traitement thermique) sous forme de cavités. Le phénomène est aussi annoncé dans les aciers austénitiques sous chargement cathodique [24].

Nous voulons tester cette hypothèse. Il faut tout d'abord générer des structures de joints de grains comportant une ségrégation d'équilibre de SAV. On évaluera leur cohésion par la suite.

\section{SIMULATION MONTÉ CARLO DE LACUNES}

Il s'agit de simulations de l'équilibre thermodynamique dans un ensemble pertinent pour le problème de fragilisation : qui permette la formation de SAV. Dans nos calculs, le système Al-H a été choisit car l'énergie de formation de la lacune est faible $(0.7 \mathrm{eV})$ et l'énergie de ségrégation importante $(-0.4 \mathrm{eV})$. En conséquence, des concentrations de lacunes élevées sont obtenues, pour des concentrations en hydrogène raisonnables ( $<1 \%$ at.). On ne simule pas la diffusion depuis la surface, mais un petit système 


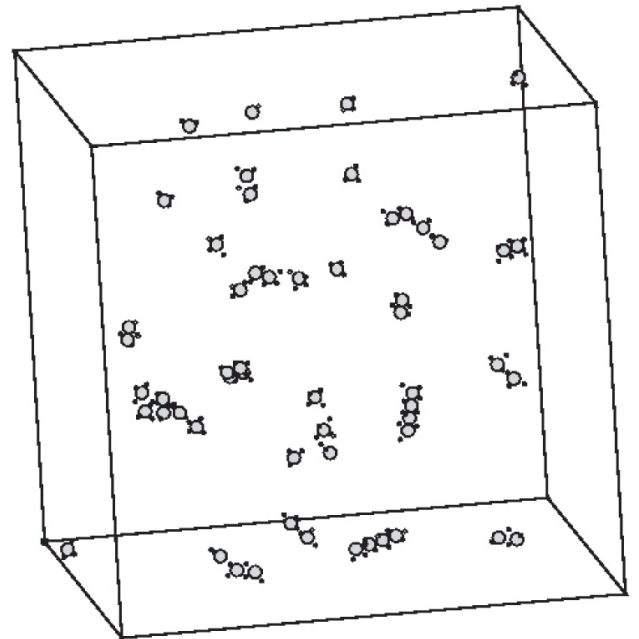

(a)

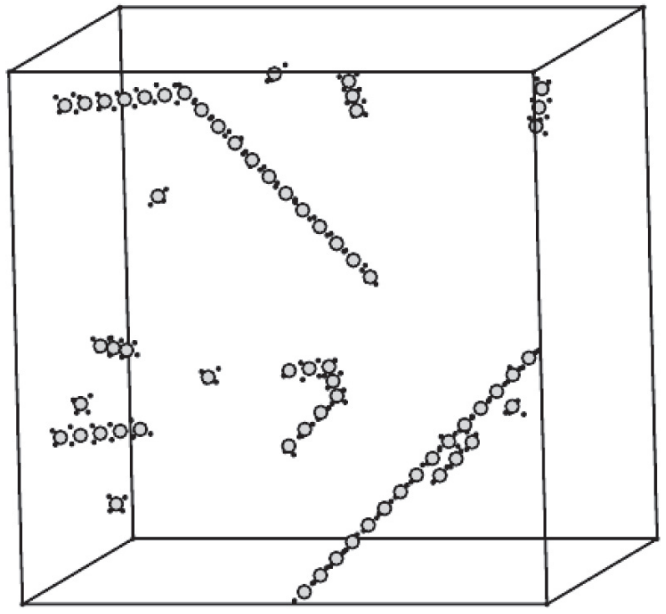

(b)

Figure 4. Deux configurations issues de simulations Monte Carlo Grand Canonique $\left(\left(\mu_{\mathrm{Al}}, \mathrm{N}_{\mathrm{H}}\right), \mathrm{V}, \mathrm{T}\right)$, avec déplacements, à $\mathrm{T}=400 \mathrm{~K}$ (a) et $\mathrm{T}=300 \mathrm{~K}(\mathrm{~b})$, pour une concentration atomique de $1 \%$ en hydrogène. Le potentiel interatomique est de type EAM pour le système Al-H. Les cercles représentent les lacunes, les petits cercles les atomes d'hydrogène, les atomes Al ne sont pas représentés. Le détail des calculs peut être trouvé dans [26].

comportant quelques dizaines de milliers de particules, à l'équilibre avec un réservoir qui impose la température et le potentiel chimique du métal. Le nombre d'atomes d'hydrogène est constant, mais le nombre d'atomes d'Al fluctue.

Les simulations Monte Carlo [25] permettent de générer une suite d'états qui peuvent être considérées comme des images du système à différents instants. Quand cette collection est très grande, chaque état microscopique y est représenté un nombre de fois qui correspond à son «poids statistique »: le facteur de Boltzmann. L'état thermodynamique est définit par $\left(\mu_{\mathrm{Al}}, \mathrm{N}_{\mathrm{H}}, \mathrm{T}, \mathrm{V}\right)$ où $\mu_{\mathrm{Al}}$ est le potentiel chimique de $\mathrm{Al}, \mathrm{N}_{\mathrm{H}}$ est le nombre d'hydrogènes dans le système, $\mathrm{T}$ la température et $\mathrm{V}$ le volume $\mathrm{du}$ système. Un état microscopique est définit par $\left(N_{A l},\{\vec{r}\}^{N_{A} l},\{\vec{r}\}^{N_{H}}\right)$, le nombre de particules d'aluminium, la position de chaque particule $\mathrm{Al}$ et $\mathrm{H}$. Pour construire la collection d'images, on propose une transition d'un état microscopique à un autre. La transition est acceptée ou rejetée selon une probabilité qui dépend de la différence d'énergie entre les deux états et de la température. L'intérêt de cette méthode est qu'il n'est pas nécessaire de déplacer les particules selon une « trajectoire » réaliste. Les transitions peuvent être choisies d'une manière qui fournisse un bon échantillonnage des états les plus importants. Ainsi, les simulations à potentiel chimique constant sont effectuées en proposant des insertions et des extractions de particules, au hasard dans le système (pas de mécanisme de diffusion), ce qui permet de créer efficacement le nombre de lacunes qui est requi par la thermodynamique [26]. D'autres types de «mouvements » sont effectués: des déplacements de particules (ce qui permet de relaxer les défauts, donc de prendre en compte les contraintes qu'ils induisent dans le système), des échanges entre différents de sites du réseau...

On peut mesurer toutes les quantités intéressantes en effectuant des moyennes d'ensemble sur les éléments de la chaînes. Par exemple : la position moyenne de chaque particule, la concentration moyenne de lacunes, la distribution moyenne des atomes d'hydrogène dans les lacunes, la probabilité de trouver deux lacunes en position de premier voisin, troisième voisin ...

La figure 4 représente deux configurations typiques de lacunes surabondantes produites par ce type de simulation. En moyenne, une lacune est occupée par deux atomes d'hydrogène et s'aligne sous forme 


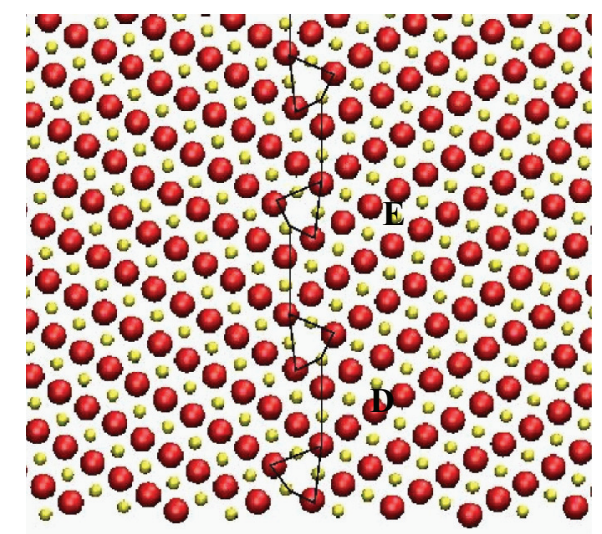

Figure 5. Joint de flexion symétrique $\Sigma=33$ (554) [110] utilisé pour le calcul de ségrégation de lacunes surabondantes. Il comporte deux unité structurales $\mathrm{D}$ (joint de macle) et $\mathrm{E}$ qui sont très différentes vis à vis de la formation de lacunes.

de chaînes le long de directions [110]. Le potentiel interatomique étant empirique, il ne faut pas prêter trop d'attention à la structure qui est obtenue, mais simplement retenir que les clusters lacune-hydrogène ont tendance à s'ordonner à basse température.

La méthode est à lors actuel étendue au joint $\Sigma=33$ (554) [110] (Fig. 5). Il présente deux unités structurales («motifs ») qui ont un comportement très différent vis à vis de la formation de lacunes. « $\mathrm{D}$ » est le motif d'une macle. Les sites qui le compose ont un environnement très proche d'un site de volume, donc on ne s'attend pas à ce qu'il y ait formation massive de lacunes surabondantes sur cette structure. Par contre E présente des sites qui ont une énergie de formation de lacunes nettement inférieure au volume. D'après les premiers résultats à $\mathrm{T}=0 \mathrm{~K}$, ce sont des sites privilégiés pour accueillir des SAV. Pour la suite de ce projet, l'idée est de choisir une concentration en hydrogène où les unités structurales E sont saturées en SAV, puis de voir comment l'ordre chimique local (les interactions entre lacunes) permettent progressivement d'augmenter les concentrations sur $\mathrm{D}$, quand $\mathrm{N}_{\mathrm{H}}$ augmente. De plus, ce joint de flexion symétrique a un plan proche de l'orientation (111), ce qui devrait permettre une comparaison intéressante avec les résultats de rupture sur monocristaux $\{111\}$. Ces calculs sont maintenant présentés.

\section{SIMULATION DYNAMIQUE MOLÉCULAIRE DE FISSURES : FRAGILITÉ/DUCTILITÉ INTRINSÈQUE}

La cohésion du cristal est déterminée en simulant la propagation d'une fissure par Dynamique Moléculaire [25] trempée. Il s'agit de résoudre numériquement les équations de Newton $\vec{f}_{i}=m_{i} \vec{\gamma}_{i}$ où $\mathrm{f}_{\mathrm{i}}$ est la force sur la particule $\mathrm{i}, \mathrm{m}_{\mathrm{i}}$ sa masse et $\gamma_{i}$ son accélération. Les forces sont calculées en prenant l'opposé du gradient de l'énergie potentielle du système vis à vis de la position de la particule. Le système est « trempé »: toutes les vitesses sont mises à zéro quand les forces sont opposées aux vitesses des particules. Les particules se déplacent, en moyenne, dans des directions qui entraînent des baisses de l'énergie du système, jusqu'à ce que les forces soient nulles sur toutes les particules. Le système est alors à l'équilibre mécanique.

Le système est une boîte parallélépipédique. L'axe de traction est selon z. Les « conditions aux bords » sont : déplacement imposé dans la direction $\mathrm{z}\left(\mathrm{U}_{\mathrm{z}}\right)$ sur les surfaces de normale $\mathrm{z}$; conditions périodiques imposées dans les deux directions transverses : déformations planes. Une fissure « mathématique » est créée, perpendiculairement à z, au milieu du système en « coupant » les liaisons chimiques qui traversent le plan de la fissure [27] dans un intervalle de longueur 2a. «2a » est la longueur de la fissure dans le problème 2D standard de mécanique des milieux continus. Le système est ensuite 


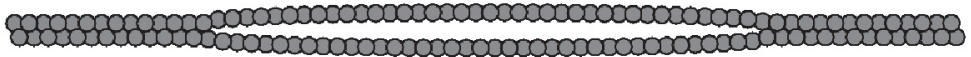

(a)

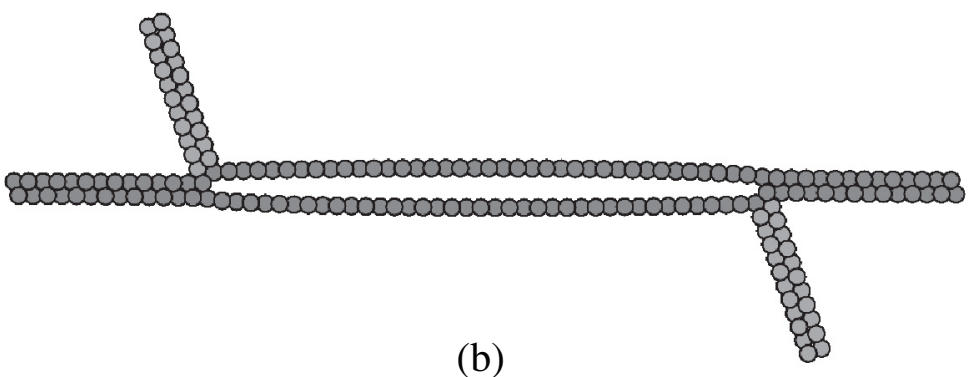

Figure 6. Fissures dans $\mathrm{Al}$ (potentiel [29]) monocristallin (le front de fissure est le long de [1-10], la direction de propagation [2-11] et la normale [111]) sollicitée en mode I.

relaxé jusqu'à ce que toutes les forces soient nulles. On peut alors définir les contraintes à l'échelle atomique de sorte que ces valeurs, quand elles sont moyennées sur une petite surface, soient cohérentes avec les contraintes de Cauchy utilisées en mécanique des milieux continus. Il s'agit des contraintes du Viriel, définies sur chaque atome.

Pour caractériser le chargement mécanique sur la fissure, on utilise les résultats de mécanique des milieux continus [28]. La solution de Inglis $T_{z z}(x)=T_{z z}^{\infty} \frac{X}{\sqrt{X^{2}-1}}$ où $X=\frac{x-X_{c}}{\tilde{a}}\left(\mathrm{X}_{\mathrm{c}}\right.$ est la position du centre de la fissure, a la demi longueur) est ajustée sur le profile de contrainte obtenu à l'échelle atomique. L'ajustement donne $T_{z z}^{\infty}$ et $\tilde{a}$ qui permettent de définir le facteur d'intensité des contraintes en mode I. Pour ce problème $: k_{I}=T_{z z}^{\infty} \sqrt{\tilde{a}}$. Toutes les mesures (cisaillement local, énergies d'adsorption...) qui dépendent de la structure de la pointe peuvent être exprimées en fonction de k, ce qui a l'avantage de faire tomber sur la même courbe des mesures effectuées sur différentes tailles de boîtes, à différents niveau de chargement mécanique et différentes longueurs de fissure.

En pratique, des séries de simulations sont effectuées en augmentant $\mathrm{U}_{z}$. A chaque valeur de $\mathrm{U}_{z}$ : la fissure est relaxée, $\mathrm{k}_{I}$ est mesuré ( $\mathrm{k}_{I}$ appliqué) et la morphologie de la fissure est analysée. Quand la formation d'une dislocation est détectée (Fig. 6b), $\mathrm{k}_{I}$ appliqué vaut $\mathrm{k}_{I e}$ : le facteur d'intensité des contraintes critique pour émettre une partielle depuis la pointe de la fissure. Pour le potentiel [29], $\mathrm{k}_{I e}$ vaut approximativement $0.26 M P a \sqrt{m}$. Nous avons aussi développé de nouvelles équations du mouvement qui permettent d'empêcher la localisation du cisaillement en pointe et donc de propager une fissure fragile virtuelle [30]. De la même manière que $\mathrm{k}_{I e}$, une série de simulations est effectuée à $\mathrm{k}$ appliqué croissant. La valeur critique de $\mathrm{k}$ pour laquelle la fissure fragile se propage est mesurée. Elle vaut vaut $k_{I c}=0.42 \mathrm{MPa} \sqrt{m}$. La valeur de $k_{\text {Griffith }}$, estimé à partir de l'énergie de formation de surface est $0.37 \mathrm{MPa} \sqrt{\mathrm{m}}$. L'accord est très bon, dans la fourchette des écarts qui sont généralement obtenus entre la description atomique et le bilan énergétique « macroscopique » de Griffith. Il faut admettre que le critère de Griffith n'est très bien défini à l'échelle atomique (la surface peut être rugueuse, la fissure peut être piégée par le réseau comme dans le silicium [31]...).

Nous avons donc le moyen de calculer les deux valeurs qui entrent dans le critère de Rice-Thomson. $\mathrm{k}_{I e}<\mathrm{k}_{I c}$ : l'aluminium est intrinsèquement ductile. En perspective, il serait intéressant d'effectuer le même type de calculs, mais en prenant en compte aussi le chargement par le soluté $(\mathrm{H})$ adsorbé sur les lèvres de la fissures. Ceci permettrait de définir, si elle existe, la valeur critique du chargement en soluté 
qui inverse le classement de $\mathrm{k}_{I e}$ et $\mathrm{k}_{I c}$, donnant une condition nécessaire à la propagation de fissure fragiles par effet d'hydrogène.

\section{CONCLUSION}

Dans cet article nous avons montré comment les simulations à l'échelle atomique peuvent contribuer à modéliser l'endommagement par l'hydrogène des joints de grains. En particulier dans les alliages $\mathrm{AlZnMg}$ et $\mathrm{AlMg}$ où il y a des observations directes, en MET, de ruptures par décohésion. En se basant sur des calculs ab initio dans le monocristal $\{111\}$ et sur des calculs d'énergies de ségrégation sur des familles de joints en EAM (pris dans la littérature), nous avons montré que l'hydrogène seul ne peut pas engendrer la perte drastique de tenue mécanique qui est observée expérimentalement. Un ingrédient microscopique supplémentaire doit être introduit. Nous proposons d'étudier l'influence des complexes lacune-hydrogène, ou « lacunes surabondantes », sur la tenue des joints. Pour cela, nous développons, d'une part, une méthode Monte Carlo où le nombre de particules est variable (ensemble Grand Canonique), d'autre part, les propriétés mécaniques sont évaluées en simulant la propagation de fissures en Dynamique Moléculaire. Les facteurs d'intensité des contraintes critiques $\mathrm{k}_{I e}$ et $\mathrm{k}_{I c}$ sont calculés, en relation avec le critère de Rice-Thomson pour la fragilité/ductilité intrinsèque des matériaux.

\section{Supports et remerciements}

Ce travail est supporté par la région Rhône-Alpes, le Centre Européen de Calcul Atomique et Moléculaire (CECAM, ENS-Lyon) et l'ANR (BLAN06-2_149056). Les auteurs remercient Olivier Hardouin-Duparc pour avoir corrigé ce manuscrit.

\section{Références}

[1] Magnin Th. «Advances in Corrosion-Deformation Interactions » Materials Science Forum, Vol. 202, Trans Tech Publications (1996).

[2] Brass, Chêne, Coudreuse, M 175 et M 176, Techniques de l'ingénieur.

[3] Bockris et al. Electrochemica Acta 16 (1971) 2169.

[4] Chateau J.P., Delafosse D. et Magnin, T. Acta Mater. 50 (2002) 1507.

[5] Chateau J.P., Delafosse D. et Magnin, T. Acta Mater. 50 (2002) 1523.

[6] Birnbaum H.K., et al. dans « Corrosion deformation interactions », édité par Thierry Magnin, European Federation of Corrosion Publications, The institute of materials 1997, p. 172.

[7] Chateau J.P., Thèse Ecole des Mines de Saint-Etienne (1999).

[8] Girardin G., Thèse Ecole des Mines de Saint-Etienne (2004).

[9] Rice J.R. and Thomson R., Phil. Mag. (1974) 73.

[10] Jiang D.E. and Carter E. Acta Mat. 52 (2004) 4801.

[11] Van der Ven, A. Ceder, G. Acta Mat. 52 (2004) 1223.

[12] Serebrinsky S. Carter et E.A. Ortiz, M. J. Mech. Phys. Solids 52 (2004) 2403.

[13] Christodoulou L. and Flower H.M. Acta Metall. 28 (1980) 481.

[14] Scamans G.M. Alani, R. Swann, P.R. Corr. Sci. 16 (1976) 443.

[15] Malis T. and Chaturvedi M.C. J. Mater. Sci. 17 (1982) 1479.

[16] Scamans G.M., J. Mater. Sci. 13 (1978) 27.

[17] Alani et R. Swann, P.R. Corros. Br. J. 12 (1977) 80.

[18] Shiga M., Yamaguchi, M. and Kaburaki, H. Phys. Rev. B 68 (2003) 245402.

[19] Fukai Y., Alloys J. Compounds 356-356 (2003) 263.

[20] Fukai Y. « The Metal-Hydrogen System », second edition, Springer (2005).

[21] Birnbaum H.K. et al. J. Alloyes Comp. 253-254 (1997) 260.

[22] Buckley, C.E. Birnbaum, H.K. J. Alloyes Comp. 330-332 (2002) 649. 
[23] Rozenak P. et al. J. Alloyes Comp. 387 (2005) 201.

[24] Gavriljuk V.G. et al. Scripta Mat. 34 (1996) 903.

[25] Frenkel D. and Smit, B. « Understanding Molecular Simulations », Academic Press (2002).

[26] Tanguy D. and Masreschal M. Phys. Rev. B 72 (2005) 174116.

[27] Cleri F., Phillpot, S.R., Wolf D. and Yip S.J., Am. Ceram. Soc., 81 (1998) 501.

[28] Mattoni A., Colombo L. and Cleri F., Phys. Rev. B 70094108 (2004).

[29] Aslanides A. and Pontikis, V. Phil. Mag. Lett. 78 (1998) 377.

[30] Tanguy, D. «Constrained Molecular Dynamics equations for quantifying intrinsic ductility versus brittleness » Phys. Rev. B 76 (2007) 144115.

[31] Pérez R. and Gumbsch, P. Phys. Rev. Lett. 84 (2000) 5347. 